\title{
The generation of strong magnetic fields during the formation of the first stars
}

\author{
Sharanya Sur*, Robi Banerjee, Ralf S. Klessen \\ Zentrum für Astronomie der Universität Heidelberg, Institut für Theoretische Astrophysik, \\ Albert-Ueberle-Str. 2, 69120 Heidelberg, Germany \\ E-mail: ssurdita.uni-heidelberg.de
}

\section{Dominik R.G. Schleicher}

Leiden Observatory, Leiden University, P.O. Box 9513, NL-2300 RA Leiden, the Netherlands; ESO, Karl-Schwarzschild-Strasse 2, 85748 Garching bei München, Germany

\section{Christoph Federrath}

Ecole Normale Supérieure de Lyon, CRAL, 69364 Lyon, France

\begin{abstract}
The ubiquity of turbulence in the primordial minihalos has been shown from cosmological hydrodynamical simulations of primordial star formation. This has strong implications on the subsequent evolution, in particular on the generation of magnetic fields via small-scale dynamo action. Using high-resolution numerical simulations, we show that in the presence of turbulence, initial weak seed magnetic fields are exponentially amplified by the small-scale dynamo during the formation of the first stars. The field amplification due to the dynamo is shown to provide additional amplification over what is expected from pure gravitational compression of the field lines. We find that the presence of the small-scale dynamo can only be identified in numerical simulations where the turbulent motions in the central collapsing core are resolved by at least 32 grid cells. We conclude that strong magnetic fields are generated during the birth of the first stars in the universe and discuss implications for cosmic evolution.
\end{abstract}

Cosmic Radiation Fields: Sources in the early Universe

November 9-12, 2010

Desy, Germany

\footnotetext{
* Speaker.
} 


\section{Motivation}

Over the course of the last decade, a great deal of progress has been made in understanding the physical processes governing the birth of the first stars and their influence on later epochs of structure formation $[1,2,3,4,5]$. These studies have ignored the possible role of magnetic fields primarily, because the initial field strengths obtained from either cosmological processes like inflation and phase transition [6,7] or astrophysical processes like the Biermann battery [8] or the Weibel instability $[9,10]$ are highly uncertain. However, recent FERMI observations [11] provide a lower limit of $10^{-15} \mathrm{G}$ for the magnetic field strength in the intergalactic medium (IGM), consistent with the predicted field strength from Biermann battery mechanism at a redshift of $z \sim 10$ [12].

Interestingly, hydrodynamical simulations starting from cosmological initial conditions and following the gravitational collapse of the primordial gas all the way down to protostellar densities, reveal the presence of turbulence in the minihalos of the first star-forming cloud. This opens the possibility of amplifying weak initial seed magnetic fields at the expense of the kinetic energy in the turbulent fluid motions, to eventually generate strong seed fields by a process commonly referred to as small-scale dynamo action (see [13] for a review). In the context of the first stars and the first galaxies, dynamo amplification of small seed fields has been previously addressed using semianalytic models [14]. In this contribution, we present the results obtained from three-dimensional numerical simulations aimed at exploring the possibility of amplifying weak initial seed magnetic fields through small-scale dynamo action during the formation of the first stars. We also discuss the implications of the dynamo generated random magnetic field on the formation process of the first stars and their subsequent evolution.

\section{Generation of strong magnetic fields during the formation of the first stars}

We focus on the gravitational collapse and magnetic field amplification of the baryon-dominated inner parts of a collapsing primordial halo. The initial conditions of our simulations motivated from the cosmological models of [1], [4] and [15] correspond to a turbulent, magnetized Bonnor-Ebert sphere. For more details on the numerical setup and initial conditions, the readers are referred to [16]. The efficiency of the dynamo process depends on the Reynolds number and is thus related to how well the turbulent motions are resolved $[17,18]$. Higher resolution yields larger field amplification. We report results obtained from five numerical simulations where we resolve $\lambda_{\mathrm{J}}$ by 8,16 , 32, 64 and 128 cells. Fig. 1 shows a snapshot of the central region of the collapsing core in our highest-resolution simulation at a time when the central density has increased by a factor of $\sim 10^{6}$. Starting from a weak $1 \mathrm{nG}$ field, the magnetic field grows by a factor of $10^{6}$, reaching a peak value of about $1 \mathrm{mG}$ in the central core at $\tau \sim 12$ as shown in Fig. 2.1a. Here, $\tau$ is a dimensionless time coordinate normalized in terms of the local free-fall time (see Eq. 2 in [16]). As shown in Fig. 2.1b, the field amplification is indeed stronger than what is expected from pure flux-freezing. This indicates that the small-scale dynamo provides significant additional amplification over compression during the birth of the first stars. Capturing the small-scale dynamo in these simulations requires a minimum resolution of 32 cells per Jeans length (see Fig. 2.2b). The radial infall motions dominate the total velocity in the envelope as shown in Fig. 2.2a. The turbulence is maintained on scales of $\lambda_{\mathrm{J}}$ and dominates the dynamics inside the core region as can be seen from Figs. 2.2b and 2.2c. The 

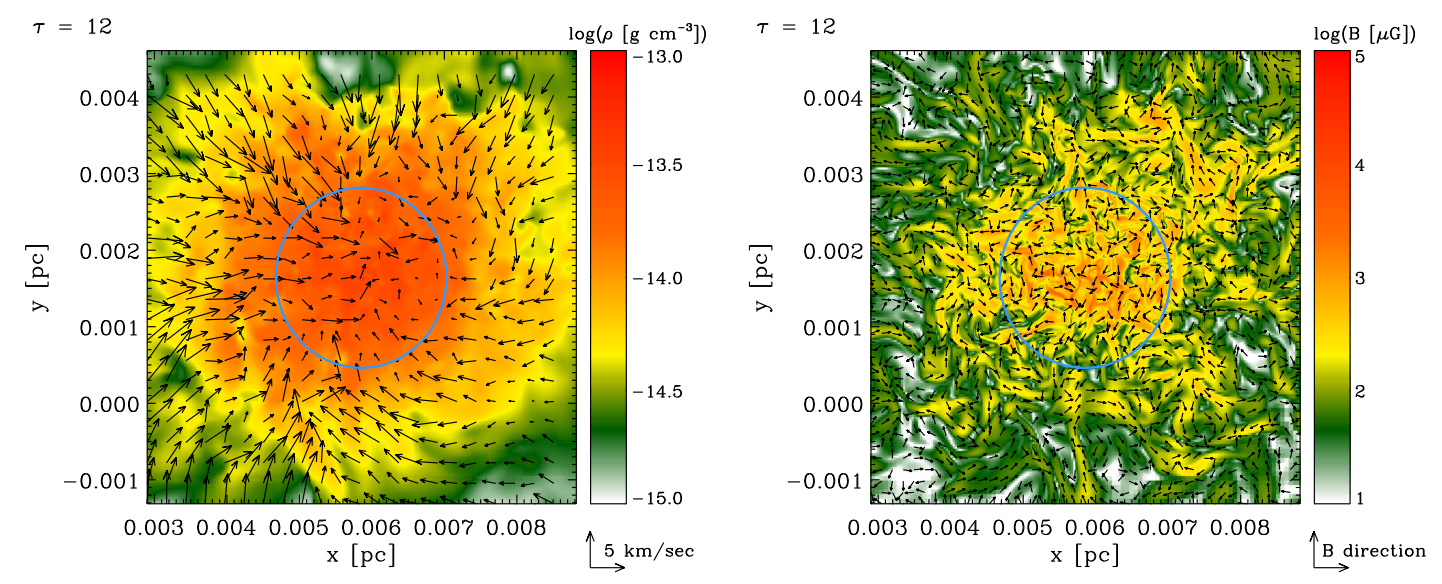

Figure 1: Two-dimensional slices through the center of the collapsing core at the time when the initial field strength has increased by a factor of $\sim 10^{6}$, showing the central region of our highest-resolution simulation ( $\lambda_{\mathrm{J}}$ resolved by 128 cells). The circle indicates the control volume $V_{\mathrm{J}}$ centered on the position of the current density peak. The left image shows the density and the velocity component in the $x y$-plane, indicating radial infall in the outer regions and turbulent motions in the inner core. The right image depicts the total magnetic field amplitude and the local magnetic field direction.

radial profile of the Mach number in Fig. 2.2d illustrates that the infalling velocities are supersonic, while inside the core, the velocities are subsonic.

\section{Conclusions and Discussions}

In summary, we conclude that the ubiquity of turbulence in the primordial minihalo can lead to the generation of strong magnetic fields by the small-scale turbulent dynamo action. We find in our highest resolution simulation that a weak seed field of $\sim 1 \mathrm{nG}$, is amplified to about $1 \mathrm{mG}$ strength in the central collapsing core during the formation of the first stars. The small-scale dynamo only works in simulations in which the turbulent motions within the local Jeans length are resolved by at least 32 cells.

The generation of strong magnetic fields has interesting consequences for our understanding of how the first stars form and how they influence subsequent cosmic evolution. Recent hydrodynamical simulations [19] suggest that the primordial gas is highly susceptible to fragmentation even for subsonic turbulence. Magnetic fields can significantly influence this scenario provided they are amplified to values close to equipartition. In particular, recent advances in the modeling of galactic star-forming clouds imply that the presence of magnetic fields can reduce the level of fragmentation [20], and by doing so strongly influences the stellar mass spectrum. To investigate the role of magnetic fields in influencing the fragmentation properties of the primordial cloud, it is necessary to evolve our existing simulations beyond the exponential growth phase. This will help us to gain an insight on the saturated field strengths to be expected in the protostellar core of the first stars. Furthermore, small-scale dynamo generated fields can influence the dynamics in the protostellar accretion disk via the magneto-rotational instability (MRI) [21, 22]. Self-gravitational instabilities in concert with the MRI can effectively decrease the accretion rate and change the disk morphology 

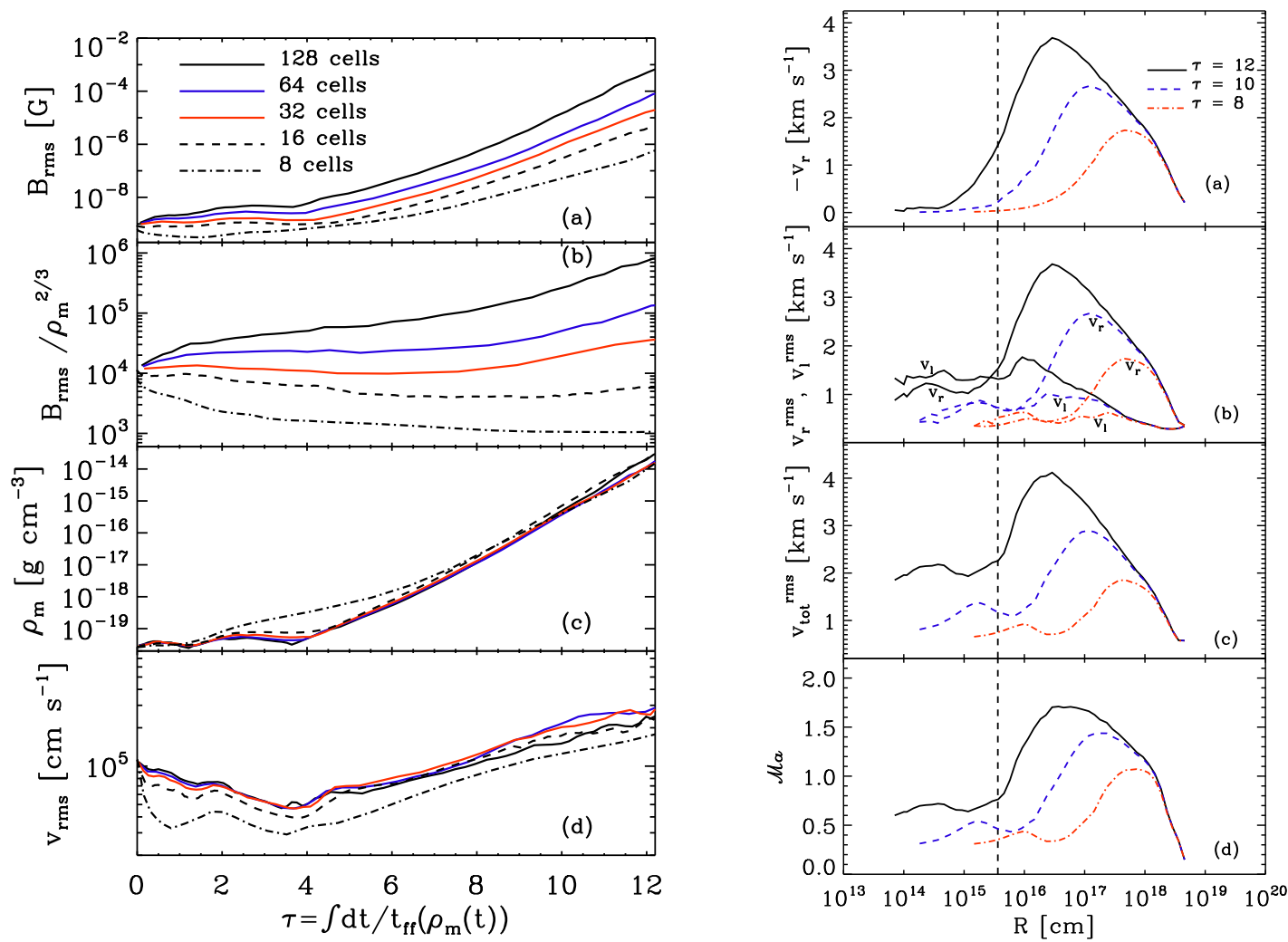

Figure 2: Left (Fig. 2.1): Evolution of the dynamical quantities in the central Jeans volume as a function of $\tau$, for five runs with different number of cells to resolve the local Jeans length. Panel (a) shows the rms magnetic field strength $B_{\mathrm{rms}}$, (b) the evolution of $B_{\mathrm{rms}} / \rho_{\mathrm{m}}^{2 / 3}$, showing the turbulent dynamo amplification by dividing out the maximum possible amplification due to perfect flux freezing, (c) the evolution of the mean density $\rho_{\mathrm{m}}$ and (d) the rms velocity $v_{\text {rms }}$. The runaway collapse commences at about $\tau \sim 4$.

Right (Fig. 2.2): Time evolution of the the radial profile of the mean value of the infall velocity, $v_{\mathrm{r}}$ (panel a), the radial profile of the rms value of $v_{\mathrm{r}}$ together with the rms fluctuations of the lateral velocity $v_{\mathrm{l}}$, i.e., the component perpendicular to $v_{\mathrm{r}}$ (panel b), the radial profile of the rms value of the total velocity (panel c) and the Mach number (panel d). The vertical line indicates the Jeans radius at $\tau=12$. The data are taken from our highest-resolution run.

[23]. The other interesting questions emanating from this study that merits a careful analysis are on what scales does the magnetic field grow during the gravitational collapse of a magnetized gas cloud, - what is/are the characteristic driving scale(s) of turbulence in a gravitationally collapsing system and what is the ratio of solenoidal to compressible motions in a gravity-driven turbulent core. Addressing these questions is crucial to develop an understanding of the complex interplay of gravity, turbulence and magnetic fields not only in the context of the first stars but also applies to any turbulent, magnetized collapsing system in general. We address these questions in more detail in [24]. 


\section{Acknowledgments}

S. S thanks the German Science Foundation (DFG) for financial support via the priority program 1177 'Witnesses of Cosmic History: Formation and evolution of black holes, galaxies and their environment' (grant KL 1358/10). D. R. G. S is supported by the European Community's Seventh Framework Programme (FP7/2007-2013) under grant agreement No 229517. R. B is funded by the Emmy-Noether grant (DFG) BA 3607/1. C. F and R. S. K are supported by the Landesstiftung Baden-Württemberg via their program International Collaboration II under grant PLS-SPII/18. Computing time at the Leibniz-Rechenzentrum in Garching (Germany) via grant No. h1221 and partial support from a Frontier grant of Heidelberg University funded by the German Excellence Initiative is acknowledged.

\section{References}

[1] T. Abel, G. L. Bryan and M. L. Norman, The Formation of the First Star in the Universe, Science, 295, 93-98 (2002)

[2] V. Bromm and R. B. Larson, The First Stars, Ann. Revs. Astron. Astrophys., 42, 79-118 (2004)

[3] B. O'Shea and M. L. Norman, Population III Star Formation in a $\Lambda$ CDM Universe. I. The Effect of Formation Redshift and Environment on Protostellar Accretion Rate, Astrophys. J., 654, 66-92, 2007

[4] N. Yoshida, K. Omukai and L. Hernquist, Protostar Formation in the Early Universe, Science, 321, 669 (2008)

[5] M. J. Turk, T. Abel and B. O'Shea, The Formation of Population III Binaries from Cosmological Initial Conditions, Science, 325, 601 (2009)

[6] D. Grasso and H. R. Rubinstein, Magnetic fields in the early Universe, Phys. Rep., 348, 163-266 (2001)

[7] K. Subramanian, Magnetic fields in the early Universe, Astronomische Nachrichten, 331, 110 (2010)

[8] L. Biermann, Über den Ursprung der Magnetfelder auf Sternen und im interstellaren Raum (miteinem Anhang von A. Schlüter), Zeitschrift Naturforschung Teil A, 5, 165 (1950)

[9] R. Schlickeiser and P. K. Shukla, Cosmological Magnetic Field Generation by the Weibel Instability, Astrophys. J., 599, L57-L60 (2003)

[10] M. V. Medvedev, L. O. Silva, M. Fiore, R. A. Fonseca and W. B. Mori, Generation of Magnetic Fields in Cosmological Shocks, Journal of Korean Astronomical Society, 37, 533-541 (2004)

[11] F. Tavecchio, G. Ghisellini, L. Foschini, G. Bonnoli, G. Ghirlanda and P. Coppi, The intergalactic magnetic field constrained by Fermi/Large Area Telescope observations of the TeV blazar 1ES0229+200, Mon. Not. Roy. Astr. Soc., 406, L70-L74 (2010)

[12] H. Xu, B. W. O'Shea, D. C. Collins, M. L. Norman, H. Li and S. Li, The Biermann Battery in Cosmological MHD Simulations of Population III Star Formation, Astrophys. J., 688, L57-L60 (2008)

[13] A. Brandenburg and K. Subramanian, Astrophysical magnetic fields and nonlinear dynamo theory, Phys. Rep., 417, 1-209 (2005) 
[14] D. R. G. Schleicher, R. Banerjee, S. Sur, T. G. Arshakian, R. S. Klessen, R. Beck and M. Spaans, Small-scale dynamo action during the formation of the first stars and galaxies. I. The ideal MHD limit, Astron. Astrophys., 522, 115 (2010)

[15] V. Bromm, P. S. Coppi and R. B. Larson, The Formation of the First Stars. I. The Primordial Star-forming Cloud, Astrophys. J., 564, 23-51 (2002)

[16] S. Sur, D. R. G. Schleicher, R. Banerjee, C. Federrath and R. S. Klessen, The Generation of Strong Magnetic Fields During the Formation of the First Stars, Astrophys. J., 721, L134-L138 (2010)

[17] N. E. Haugen, A. Brandenburg and W. Dobler, Simulations of nonhelical hydromagnetic turbulence, Phys. Rev. E, 70, 016308 (2004)

[18] D. S. Balsara, J. Kim, M. -M. Mac Low and G. J. Mathews, Amplification of Interstellar Magnetic Fields by Supernova-driven Turbulence, Astrophys. J., 617, 339-349 (2004)

[19] P. C. Clark, S. C. O Glover, R. S. Klessen and V. Bromm, Gravitational fragmentation in turbulent primordial gas and the initial mass function of Population III stars, Astrophys. J., 727, doi:10.1088/0004-637X/727/2/110(2011)

[20] P. Hennebelle and R. Teyssier, Magnetic processes in a collapsing dense core. II. Fragmentation. Is there a fragmentation crisis?, Astron. Astrophys., 477, 25-34 (2008)

[21] J. Tan and E. Blackman, Protostellar Disk Dynamos and Hydromagnetic Outflows in Primordial Star Formation, Astrophys. J., 603, 401-413 (2004)

[22] J. Silk and M. Langer, On the first generation of stars, Mon. Not. Roy. Astr. Soc., 371, 444-450 (2006)

[23] S. Fromang, S. A. Balbus, C. Terquem and J. -P. DeVilliers, Evolution of Self-Gravitating Magnetized Disks. II. Interaction between Magnetohydrodynamic Turbulence and Gravitational Instabilities, Astrophys. J., 616, 364-375 (2004)

[24] C. Federrath, S. Sur, D. R. G. Schleicher, R. Banerjee and R. S. Klessen, A new Jeans resolution criterion for $(M) H D$ simulations of self-gravitating gas: Application to magnetic field amplification by gravity-driven turbulence, Astrophys. J., accepted, arXiv:1102.0266 (2011) 